CERN-PH-TH/2004-086

HEPHY-PUB 790/04

IISC-CHEP/7/04

FI2004-15

hep-ph/0405167

\title{
Fermion polarization in sfermion decays as a probe of CP phases in the MSSM
}

\author{
Thomas Gajdosik ${ }^{1}$, Rohini M. Godbole ${ }^{2}$, Sabine Kraml ${ }^{3,4}$ \\ 1 Institute of Physics, Vilnius LT-2600, Lithuania \\ 2 Centre for High Energy Physics, Indian Institute of Science, Bangalore 560012, India \\ 3 Inst. f. Hochenergiephysik, Österr. Akademie d. Wissenschaften, 1050 Vienna, Austria \\ 4 Department of Physics, CERN, Theory Division, 1211 Geneva 23, Switzerland
}

\begin{abstract}
The longitudinal polarization of fermions (tops and taus) produced in sfermion decays to neutralinos or charginos can be a useful tool for the determination of SUSY parameters. We discuss this fermion polarization in the context of the MSSM with complex parameters. We show that the dependence on CP-violating phases can be large and that the fermion polarization may hence be used as a sensitive probe of CP phases in the MSSM.
\end{abstract}

\section{Introduction}

CP violation, initially observed [1] only in the $K_{0}-\bar{K}_{0}$ system, is one feature of the Standard Model (SM) that still defies clear theoretical understanding. The CKM picture, which describes all the observed $\mathrm{CP}$ violation in terms of a single phase in the quark-mixing matrix, has been vindicated by the recent measurements of $B_{0}-\bar{B}_{0}$ mixing at BELLE and BABAR [2]. CP violation is in fact one of the necessary ingredients for generating the observed excess of baryons over antibaryons in the Universe $[3,4]$. The amount of CP violation present in the quark sector is, however, too small to generate a baryon asymmetry of the observed level of $N_{B} / N_{\gamma} \simeq$ $6.1 \times 10^{-10}[5]$. New sources of CP violation beyond the SM are therefore a necessity [6].

Supersymmetry (SUSY) is arguably the most attractive extension of the SM, as it solves, for instance, the problem of the instability of the electroweak symmetry-breaking scale against radiative corrections. Already the Minimal Supersymmetric Standard Model (MSSM) [7] provides possible new sources of $\mathrm{CP}$ violation through additional $\mathrm{CP}$-violating phases, which cannot be rotated away by simple field redefinitions. A large number of these phases, particularly those involving sparticles of the first and to a large extent of the second generation, are severely constrained by measurements of the electric dipole moments (EDMs) of the electron, muon, neutron as well as ${ }^{199} \mathrm{Hg}$ and ${ }^{205} \mathrm{Tl}$. However, these constraints are model-dependent. It has been demonstrated [8-12] that cancellations among different diagrams allow certain combinations of these phases to be large in a general MSSM. Furthermore, if the sfermions of the first 
two generations are sufficiently heavy, above the $1 \mathrm{TeV}$ range, the EDM constraints on the phase of the higgsino mass parameter $\mu=|\mu| e^{i \phi_{\mu}}$, in general constrained to $\phi_{\mu} \lesssim 10^{-2}$, get weaker; the sfermions of the third generation can still be light. Non-vanishing phases of $\mu$ and/or the trilinear scalar couplings $A_{t, b}$ can induce explicit CP violation in the Higgs sector via loop corrections [13-16]. Though these phases generate EDMs independently of the first two generations of sfermions, the EDMs are suppressed by the mass scale of the two heavy Higgses [17,18]. For a thorough discussion of the EDMs see [19] and references therein. The above mentioned phases can also have a significant influence on the Higgs production rates in the gluon fusion mode at the Tevatron and the LHC [20,21]. MSSM CP phases can hence change the Higgs phenomenology at colliders quite substantially.

All this makes the MSSM with CP-violating phases a very attractive proposition. It has therefore been the subject of many recent investigations, studying the implications of these phases on neutralino/chargino production and decay [22-33], on the third generation of sfermions [34-39], as well as the neutral [40-44] and charged [45] Higgs sector. Various CPeven and CP-odd (T-odd) observables, which can give information on these phases, have been identified. It is interesting to note that CP-even observables such as masses, branching ratios, cross sections, etc., often afford more precise probes thanks to the larger magnitude of the effects. For direct evidence of CP violation, however, CP-odd/T-odd observables as discussed e.g. in $[23,29-33,37,42]$ have to be measured.

The latest study of the $\tilde{t}, \tilde{b}$ sector in [39] demonstrates that it may be possible to determine the real and imaginary parts of $A_{t}$ to a precision of $2-3 \%$ from a fit of the MSSM Lagrange parameters to masses, cross sections and branching ratios at a future $e^{+} e^{-}$Linear Collider (LC). This requires that both the $\tilde{t}_{1,2}, \tilde{b}_{1,2}$ mass eigenstates can be produced at the LC and the branching ratios measured with high precision. In the $\tilde{\tau} / \tilde{\nu}_{\tau}$ sector $[35,36]$ the precision on $A_{\tau}$ is worse, around $10-20 \%$ for low $\tan \beta$ and about $3-7 \%$ for large $\tan \beta$.

In this paper, we show that the longitudinal polarization of fermions produced in sfermion decays, i.e. $\tilde{f} \rightarrow f \tilde{\chi}^{0}$ and $\tilde{f} \rightarrow f^{\prime} \tilde{\chi}^{ \pm}$with $f(\tilde{f})$ a third generation (s)quark or (s)lepton, can also be used as a probe of CP phases. The fermion polarization can give complementary information to the decay branching ratios and will in particular be useful if the branching ratios cannot be measured with high enough precision or if one decay channel dominates.

The average polarization of fermions produced in sfermion decays carries information on the $\tilde{f}_{L}-\tilde{f}_{R}$ mixing as well as on the gaugino-higgsino mixing [46]. The polarizations that can be measured are those of top and tau; both can be inferred from the decay lepton distributions. It is its large mass that causes the $t$ to decay before hadronization and thus the decay products can carry information about its polarization. For taus, also the energy distribution of the decay pions can be used. The polarization of the decay fermions has been used for studies of MSSM parameter determination in the CP-conserving case in [46-48]. For the CP-violating case, the phase dependence of the longitudinal fermion polarization has been mentioned in [36]. We extend these studies by discussing in detail the sensitivity of the fermion polarization to CP-violating phases in the MSSM.

The paper is organized as follows: in Section 2, we summarize our notation for the description of the sfermion, neutralino and chargino systems in the MSSM with CP violation. In Section 3, we discuss fermion polarization in sfermion decays to neutralinos, $\tilde{f} \rightarrow f \tilde{\chi}^{0}$ with $f=t, \tau$. We present numerical results on the polarization as a function of different MSSM parameters and discuss the sensitivity to CP-violating phases in the sfermion and neutralino sectors. In Section 4 we perform an analogous analysis for $\tilde{f} \rightarrow f^{\prime} \tilde{\chi}^{ \pm}$decays. In Section 5 we summarize the results and present our conclusions. 


\section{Notation and conventions}

\subsection{Sfermion system}

Ignoring intergenerational mixing, the sfermion mass matrices can be written as a series of $2 \times 2$ matrices, each of which describes sfermions of a specific flavour:

$$
\mathcal{M}_{\tilde{f}}^{2}=\left(\begin{array}{cc}
m_{\tilde{f}_{L}}^{2} & a_{f}^{*} m_{f} \\
a_{f} m_{f} & m_{\tilde{f}_{R}}^{2}
\end{array}\right)=\left(R^{\tilde{f}}\right)^{\dagger}\left(\begin{array}{cc}
m_{\tilde{f}_{1}}^{2} & 0 \\
0 & m_{\tilde{f}_{2}}^{2}
\end{array}\right) R^{\tilde{f}}
$$

with

$$
\begin{aligned}
m_{\tilde{f}_{L}}^{2} & =M_{\tilde{L}}^{2}+m_{Z}^{2} \cos 2 \beta\left(I_{3 L}^{f}-e_{f} \sin ^{2} \theta_{W}\right)+m_{f}^{2}, \\
m_{\tilde{f}_{R}}^{2} & =M_{\tilde{R}}^{2}+e_{f} m_{Z}^{2} \cos 2 \beta \sin ^{2} \theta_{W}+m_{f}^{2}, \\
a_{f} & =A_{f}-\mu^{*}\{\cot \beta, \tan \beta\}=\left|a_{f}\right| e^{i \varphi_{\tilde{f}}},
\end{aligned}
$$

for $\{$ up, down $\}$-type sfermions; $m_{f}, e_{f}$ and $I_{3}^{f}$ are the mass, electric charge and the third component of the weak isospin of the partner fermion, respectively; $M_{\tilde{L}}, M_{\tilde{R}}$ and $A_{f}$ are soft SUSY-breaking parameters for each family, and $\mu$ is the higgsino mass parameter; $A_{f}$ and $\mu$ can have complex phases: $A_{f}=\left|A_{f}\right| e^{i \phi_{A_{f}}}$ and $\mu=|\mu| e^{i \phi_{\mu}}$.

According to eq. (1), $\mathcal{M}_{\tilde{f}}^{2}$ is diagonalized by a unitary rotation matrix $R^{\tilde{f}}$. The weak eigenstates $\tilde{f}_{L}$ and $\tilde{f}_{R}$ are thus related to their mass eigenstates $\tilde{f}_{1}$ and $\tilde{f}_{2}$ by

$$
\left(\begin{array}{c}
\tilde{f}_{1} \\
\tilde{f}_{2}
\end{array}\right)=R^{\tilde{f}}\left(\begin{array}{c}
\tilde{f}_{L} \\
\tilde{f}_{R}
\end{array}\right), \quad R^{\tilde{f}}=\left(\begin{array}{cc}
\cos \theta_{\tilde{f}} e^{i \varphi_{\tilde{f}}} & \sin \theta_{\tilde{f}} \\
-\sin \theta_{\tilde{f}} & \cos \theta_{\tilde{f}} e^{-i \varphi_{\tilde{f}}}
\end{array}\right)
$$

with $\theta_{\tilde{f}}$ and $\varphi_{\tilde{f}}=\operatorname{Arg}\left(a_{f}\right)$ the sfermion mixing angle and phase. Since the off-diagonal element of $\mathcal{M}_{\tilde{f}}^{2}$ is proportional to $m_{f}$, this mixing is mostly relevant to the third generation, $\tilde{f}=\tilde{t}, \tilde{b}, \tilde{\tau}$, on which we concentrate in the following. The mass eigenvalues are given by

$$
m_{\tilde{f}_{1,2}}^{2}=\frac{1}{2}\left(m_{\tilde{f}_{L}}^{2}+m_{\tilde{f}_{R}}^{2} \mp \sqrt{\left(m_{\tilde{f}_{L}}^{2}-m_{\tilde{f}_{R}}^{2}\right)^{2}+4\left|a_{f} m_{f}\right|^{2}}\right) .
$$

By convention, we choose $\tilde{f}_{1}$ to be the lighter mass eigenstate, $m_{\tilde{f}_{1}} \leq m_{\tilde{f}_{2}}$. Notice also that $m_{\tilde{f}_{1}} \leq m_{\tilde{f}_{L, R}} \leq m_{\tilde{f}_{2}}$. For the mixing angle $\theta_{\tilde{f}}$ we choose

$$
\cos \theta_{\tilde{f}}=\frac{-\left|a_{f} m_{f}\right|}{\sqrt{\left(m_{\tilde{f}_{L}}^{2}-m_{\tilde{f}_{1}}^{2}\right)^{2}+\left|a_{f} m_{f}\right|^{2}}}, \quad \sin \theta_{\tilde{f}}=\frac{m_{\tilde{f}_{L}}^{2}-m_{\tilde{f}_{1}}^{2}}{\sqrt{\left(m_{\tilde{f}_{L}}^{2}-m_{\tilde{f}_{1}}^{2}\right)^{2}+\left|a_{f} m_{f}\right|^{2}}},
$$

which places $\theta_{\tilde{f}}$ in the 2 nd quadrant of the unit circle. The $\tilde{f}_{L}-\tilde{f}_{R}$ mixing is large if $\mid m_{\tilde{f}_{L}}^{2}-$ $m_{\tilde{f}_{R}}^{2}|\lesssim| a_{f} m_{f} \mid$, with $\left|\cos \theta_{\tilde{f}}\right|>\frac{1}{\sqrt{2}}$ if $m_{\tilde{f}_{L}}<m_{\tilde{f}_{R}}$ and $\left|\cos \theta_{\tilde{f}}\right|<\frac{1}{\sqrt{2}}$ if $m_{\tilde{f}_{R}}<m_{\tilde{f}_{L}}$. Moreover, we see that the phase dependence of $m_{\tilde{f}_{1,2}}^{2}$ and $R^{\tilde{f}}$ is determined by $\cos \left(\phi_{A_{f}}+\phi_{\mu}\right)$. This dependence is strongest if $\left|A_{f}\right| \simeq|\mu|\{\cot \beta, \tan \beta\}$. This issue will be discussed in more detail in the numerical analyses of Sections 3 and 4 . 


\subsection{Neutralino system}

In the basis

$$
\Psi_{j}^{0}=\left(-i \lambda^{\prime},-i \lambda^{3}, \psi_{H_{1}}^{0}, \psi_{H_{2}}^{0}\right)
$$

the neutralino mass matrix is:

$$
\mathcal{M}_{N}=\left(\begin{array}{cccc}
M_{1} & 0 & -m_{Z} \sin \theta_{W} \cos \beta & m_{Z} \sin \theta_{W} \sin \beta \\
0 & M_{2} & m_{Z} \cos \theta_{W} \cos \beta & -m_{Z} \cos \theta_{W} \sin \beta \\
-m_{Z} \sin \theta_{W} \cos \beta & m_{Z} \cos \theta_{W} \cos \beta & 0 & -\mu \\
m_{Z} \sin \theta_{W} \sin \beta & -m_{Z} \cos \theta_{W} \sin \beta & -\mu & 0
\end{array}\right) .
$$

The gaugino mass parameters $M_{1,2}$ and the higgsino mass parameter $\mu$ can in principle all be complex. The phase of $M_{2}$ can be rotated away, which leaves us with two phases in this sector: $\phi_{1}$, the phase of $M_{1}$, and $\phi_{\mu}$, the phase of $\mu$.

The matrix of eq. (9) is diagonalized by the unitary mixing matrix $N$ :

$$
N^{*} \mathcal{M}_{N} N^{\dagger}=\operatorname{diag}\left(m_{\tilde{\chi}_{1}^{0}}, m_{\tilde{\chi}_{2}^{0}}, m_{\tilde{\chi}_{3}^{0}}, m_{\tilde{\chi}_{4}^{0}}\right) \text {, }
$$

where $m_{\tilde{\chi}_{n}^{0}}, n=1, \ldots, 4$, are the (non-negative) masses of the physical neutralino states. We choose the ordering $m_{\tilde{\chi}_{1}^{0}}<\ldots<m_{\tilde{\chi}_{4}^{0}}$. A concise discussion of the neutralino sector with complex phases can be found in [33].

\subsection{Chargino system}

The chargino mass matrix is:

$$
\mathcal{M}_{C}=\left(\begin{array}{cc}
M_{2} & \sqrt{2} m_{W} \sin \beta \\
\sqrt{2} m_{W} \cos \beta & \mu
\end{array}\right) .
$$

It is diagonalized by the two unitary matrices $U$ and $V$,

$$
U^{*} \mathcal{M}_{C} V^{\dagger}=\operatorname{diag}\left(m_{\tilde{\chi}_{1}^{ \pm}}, m_{\tilde{\chi}_{2}^{ \pm}}\right)
$$

where $m_{\tilde{\chi}_{1,2}^{ \pm}}$are the masses of the physical chargino states with $m_{\tilde{\chi}_{1}^{ \pm}}<m_{\tilde{\chi}_{2}^{ \pm}}$.

\section{Fermion polarization in $\tilde{f} \rightarrow f \tilde{\chi}^{0}$ decays}

The sfermion interaction with neutralinos is $(i=1,2 ; n=1, \ldots, 4)$

$$
\begin{aligned}
\mathcal{L}_{f \tilde{f} \tilde{\chi}^{0}} & =g \bar{f}\left(f_{L n}^{\tilde{f}} P_{R}+h_{L n}^{\tilde{f}} P_{L}\right) \tilde{\chi}_{n}^{0} \tilde{f}_{L}+g \bar{f}\left(h_{R n}^{\tilde{f}} P_{R}+f_{R n}^{\tilde{f}} P_{L}\right) \tilde{\chi}_{n}^{0} \tilde{f}_{R}+\text { h.c. } \\
& =g \bar{f}\left(a_{i n}^{\tilde{f}} P_{R}+b_{i n}^{\tilde{f}} P_{L}\right) \tilde{\chi}_{n}^{0} \tilde{f}_{i}+\text { h.c. }
\end{aligned}
$$

where

$$
\begin{aligned}
a_{i n}^{\tilde{f}} & =f_{L n}^{\tilde{f}} R_{i 1}^{\tilde{f} *}+h_{R n}^{\tilde{f}} R_{i 2}^{\tilde{f} *}, \\
b_{i n}^{\tilde{f}} & =h_{L n}^{\tilde{f}} R_{i 1}^{\tilde{f} *}+f_{R n}^{\tilde{f}} R_{i 2}^{\tilde{f} *} .
\end{aligned}
$$


The $f_{L, R}^{\tilde{f}}$ and $h_{L, R}^{\tilde{f}}$ couplings are

$$
\begin{array}{ll}
f_{L n}^{\tilde{t}}=-\frac{1}{\sqrt{2}}\left(N_{n 2}+\frac{1}{3} \tan \theta_{W} N_{n 1}\right), & f_{L n}^{\tilde{b}}=\frac{1}{\sqrt{2}}\left(N_{n 2}-\frac{1}{3} \tan \theta_{W} N_{n 1}\right), \\
f_{R n}^{\tilde{t}}=\frac{2 \sqrt{2}}{3} \tan \theta_{W} N_{n 1}^{*}, & f_{R n}^{\tilde{b}}=-\frac{\sqrt{2}}{3} \tan \theta_{W} N_{n 1}^{*}, \\
h_{R n}^{\tilde{t}}=-h_{t} N_{n 4}=h_{L n}^{\tilde{t} *}, & h_{R n}^{\tilde{b}}=-h_{b} N_{k 3}=h_{L n}^{\tilde{b} *}
\end{array}
$$

for stops and sbottoms, and

$$
\begin{aligned}
& f_{L n}^{\tilde{\tau}}=\frac{1}{\sqrt{2}}\left(\tan \theta_{W} N_{n 1}+N_{n 2}\right), \\
& f_{R n}^{\tilde{\tau}}=-\sqrt{2} \tan \theta_{W} N_{n 1}^{*}, \\
& h_{R n}^{\tilde{\tau}}=-h_{\tau} N_{n 3}=h_{L n}^{\tilde{\tau} *}
\end{aligned}
$$

for staus, with the Yukawa couplings $h_{f}$ given by

$$
h_{t}=\frac{m_{t}}{\sqrt{2} m_{W} \sin \beta}, \quad h_{b, \tau}=\frac{m_{b, \tau}}{\sqrt{2} m_{W} \cos \beta} .
$$

The gaugino interaction conserves the helicity of the sfermion while the higgsino interaction flips it. In the limit $m_{f} \ll m_{\tilde{f}_{i}}$, the average polarization of the fermion coming from the $\tilde{f}_{i} \rightarrow f \tilde{\chi}_{n}^{0}$ decay can therefore be calculated as [46]

$$
\mathcal{P}_{f}=\frac{B r\left(\tilde{f}_{i} \rightarrow \tilde{\chi}_{n}^{0} f_{R}\right)-\operatorname{Br}\left(\tilde{f}_{i} \rightarrow \tilde{\chi}_{n}^{0} f_{L}\right)}{\operatorname{Br}\left(\tilde{f}_{i} \rightarrow \tilde{\chi}_{n}^{0} f_{R}\right)+\operatorname{Br}\left(\tilde{f}_{i} \rightarrow \tilde{\chi}_{n}^{0} f_{L}\right)}=\frac{\left|b_{i n}^{\tilde{f}}\right|^{2}-\left|a_{i n}^{\tilde{f}}\right|^{2}}{\left|b_{i n}^{\tilde{f}}\right|^{2}+\left|a_{i n}^{\tilde{f}}\right|^{2}}
$$

Using eqs. (14), (15) and (5) as well as $h_{L n}=h_{R n}^{*}$, we obtain, for the $\tilde{f}_{1} \rightarrow f \tilde{\chi}_{n}^{0}$ decay (omitting the overall factor $g^{2}$ and dropping the sfermion and neutralino indices for simplicity):

$$
\begin{aligned}
\left|b_{1 n}\right|^{2}-\left|a_{1 n}\right|^{2}= & \left|h_{L} \cos \theta e^{-i \varphi}+f_{R} \sin \theta\right|^{2}-\left|f_{L} \cos \theta e^{-i \varphi}+h_{L}^{*} \sin \theta\right|^{2} \\
= & \left(\left|h_{L}\right|^{2}-\left|f_{L}\right|^{2}\right) \cos ^{2} \theta-\left(\left|h_{L}\right|^{2}-\left|f_{R}\right|^{2}\right) \sin ^{2} \theta \\
& +\sin 2 \theta\left[\mathcal{R} e\left(f_{R}-f_{L}\right)\left(\mathcal{R} e h_{L} \cos \varphi+\mathcal{I} m h_{L} \sin \varphi\right)\right. \\
& \left.\quad+\mathcal{I} m\left(f_{R}+f_{L}\right)\left(\mathcal{I} m h_{L} \cos \varphi-\mathcal{R} e h_{L} \sin \varphi\right)\right] .
\end{aligned}
$$

We see that the phase dependence of $\mathcal{P}_{f}$ is the largest for maximal sfermion mixing $\left(\theta_{\tilde{f}}=3 \pi / 4\right)$ and if the neutralino has both sizeable gaugino and higgsino components. It is, moreover, enhanced if the Yukawa coupling $h_{f}$ is large. Furthermore, $\mathcal{P}_{f}$ is sensitive to $\mathrm{CP}$ violation even if just one phase, in either the neutralino or the sfermion sector, is non-zero. In particular, if only $A_{f}$ and thus only the sfermion mixing matrix has a non-zero phase, the phase-dependent term becomes

$$
\left|b_{1 n}\right|^{2}-\left|a_{1 n}\right|^{2} \stackrel{\phi_{1}=\phi_{\mu}=0}{\sim} h_{L}\left(f_{L}-f_{R}\right) \sin 2 \theta \cos \varphi
$$

If, on the other hand, only $\phi_{1}$ is non-zero we get

$$
\left|b_{1 n}\right|^{2}-\left|a_{1 n}\right|^{2} \stackrel{\phi_{A}=\phi_{\mu}=0}{\sim}\left[\mathcal{R} e h_{L} \mathcal{R} e\left(f_{R}-f_{L}\right)+\mathcal{I} m h_{L} \mathcal{I} m\left(f_{R}+f_{L}\right)\right] \sin 2 \theta .
$$

The polarization $\mathcal{P}_{f}$, eq. (23), depends only on couplings but not on masses. For the numerical analysis we therefore use $M_{1}, M_{2}, \mu, \tan \beta, \theta_{\tilde{f}}$ and $\varphi_{\tilde{f}}$ as input parameters, assuming $\phi_{\mu} \approx 0$ 
to satisfy EDM constraints more easily: assuming cancellations for the 1-loop contributions and the CP-odd Higgs mass parameter $m_{A}>300 \mathrm{GeV}, 1$-loop and 2-loop contributions to the electron EDM (eEDM), as well as their sum, stay below the experimental limit. We use the formulae of [17] for the 2-loop contributions. In order not to vary too many parameters, we use, moreover, the GUT relation $\left|M_{1}\right|=\frac{5}{3} \tan ^{2} \theta_{W} M_{2}$ and choose $\tan \beta=10$ and $\theta_{\tilde{t}}=\theta_{\tilde{\tau}}=130^{\circ}$ (large but not maximal mixing) throughout this section. The free parameters in our analysis are thus $M_{2},|\mu|$, and the phases $\phi_{1}, \varphi_{\tilde{f}}$.

Before we present the numerical results, a comment is in order: $\mathrm{CP}$ violation in the neutralino sector is determined by the phases of $M_{1}$ and $\mu$, while $\varphi_{\tilde{f}}$ originates from relative phases of $A_{f}$ and $\mu$. For stops the mixing is dominated by $A_{t}$, while for sbottoms and staus it is dominated by $\mu \tan \beta$; quite generally we have $\varphi_{\tilde{t}} \sim \phi_{A_{t}}$ unless $|\mu| \sim\left|A_{t}\right| \tan \beta$, and $\varphi_{\tilde{b}, \tilde{\tau}} \sim-\phi_{\mu}$ unless $\left|A_{b, \tau}\right| \sim|\mu| \tan \beta$. More precisely,

$$
\tan \varphi_{\tilde{f}}=\frac{x_{f} \sin \phi_{A_{f}}+\sin \phi_{\mu}}{x_{f} \cos \phi_{A_{f}}-\cos \phi_{\mu}} \quad \text { with } \quad x_{t}=\frac{\left|A_{t}\right| \tan \beta}{|\mu|}, \quad x_{b, \tau}=\frac{\left|A_{b, \tau}\right|}{|\mu| \tan \beta} .
$$

For $x_{f}>1$, any $\varphi_{\tilde{f}}$ can be reached by an appropriate choice of $\phi_{A_{f}}$, independent of $\phi_{\mu}$. For $x_{f} \leq$ 1 , however, $\varphi_{\tilde{f}}$ is restricted by $\phi_{\mu}$. In the special case of $x_{f}=1$ and $\phi_{\mu}=0, \varphi_{\tilde{f}}=\left(\phi_{A_{f}}+\pi\right) / 2$. In the stop sector this is not a problem since $x_{t}>1$ can in general be easily achieved. For sbottoms and staus, choosing $\varphi_{\tilde{b}, \tilde{\tau}}$ freely leads, however, to quite large $\left|A_{b, \tau}\right| \sim \mathcal{O}(|\mu| \tan \beta)$, which may in some cases create problems with charge- or colour-breaking minima.

Figure 1 shows the average tau polarization in $\tilde{\tau}_{1} \rightarrow \tau \tilde{\chi}_{1}^{0}$ decays as functions of $M_{2}$ and $|\mu|$ for $\tan \beta=10, \theta_{\tilde{\tau}}=130^{\circ}$ and various choices of $\phi_{1}$ and $\varphi_{\tilde{\tau}}$. The lower limits of $M_{2}$ and $|\mu|$ are given by the LEP2 constraint of $m_{\tilde{\chi}_{1}^{ \pm}}>103 \mathrm{GeV}$ [49], which automatically takes care of all other LEP constraints on the gaugino-higgsino sector. As can be seen, $\mathcal{P}_{\tau}$ is quite sensitive to $\mathrm{CP}$ phases for $|\mu|<M_{2}$, that is if the $\tilde{\chi}_{1}^{0}$ has a sizeable higgsino component. Analogously, Fig. 2 shows the average top polarization in $\tilde{t}_{1} \rightarrow t \tilde{\chi}_{1}^{0}$ decays. We observe again a strong dependence on the $\mathrm{CP}$ phases if the neutralino has a sizeable higgsino component. Unlike the case of $\mathcal{P}_{\tau}$, for $\mathcal{P}_{t}$ the dependence is still significant when $|\mu| \sim M_{2}$. We also note that some phase combinations lead to very similar polarizations, e.g. $\mathcal{P}_{t}\left(\phi_{1}=0, \varphi_{\tilde{t}}=0\right) \sim \mathcal{P}_{t}\left(\phi_{1}=\frac{\pi}{2}, \varphi_{\tilde{t}}=\frac{\pi}{2}\right)$ and $\mathcal{P}_{t}\left(\phi_{1}=0, \varphi_{\tilde{t}}=\frac{\pi}{2}\right) \simeq \mathcal{P}_{t}\left(\phi_{1}=\frac{\pi}{2}, \varphi_{\tilde{t}}=0\right)$. At a future $e^{+} e^{-}$linear collider (LC), one expects to be able to measure the tau polarization to about $3-5 \%$ and the top polarization to about $10 \%$ [48]. We see from Figs. 1 and 2 that the effects of CP-violating phases may well be visible in $\mathcal{P}_{t}$ and/or $\mathcal{P}_{\tau}$, provided $\mu$ is not too large.

We next choose specific values of $M_{2}$ and $|\mu|$ to discuss the phase dependences in more detail. Figure $3 \mathrm{a}$ shows $\mathcal{P}_{\tau}$ as a function of $\phi_{1}$, for $M_{2}=380 \mathrm{GeV},|\mu|=125 \mathrm{GeV}$ and $\varphi_{\tilde{\tau}}=0, \frac{\pi}{2},-\frac{\pi}{2}$ and $\pi$. Since for fixed $M_{2}$ and $|\mu|$ the $\tilde{\chi}_{1}^{0}$ mass changes with $\phi_{1}$, we show in addition in Fig. $3 \mathrm{~b} \mathcal{P}_{\tau}$ as a function of $\varphi_{\tilde{\tau}}$ for various values of $\phi_{1}$, with $|\mu|=125 \mathrm{GeV}$ and $M_{2}$ adjusted such that $m_{\tilde{\chi}_{1}^{0}}=100 \mathrm{GeV} . \mathcal{P}_{\tau}$ varies over a large range depending on $\phi_{1}$ and $\varphi_{\tilde{\tau}}$; if the neutralino mass parameters, $\tan \beta$ and $\theta_{\tilde{\tau}}$ are known, $\mathcal{P}_{\tau}$ can hence be used as a sensitive probe of these phases (although additional information will be necessary to resolve ambiguities and actually determine the various phases). At a LC, the parameters of the neutralino/chargino sector and also sfermion masses and mixing angles can be determined very precisely, exploiting tunable beam energy and beam polarization [50]. The actual precision depends of course on the specific scenario. To illustrate the influence of uncertainties in the knowledge of the model parameters, we take the case of $M_{2}=380 \mathrm{GeV},|\mu|=125 \mathrm{GeV}$ and 

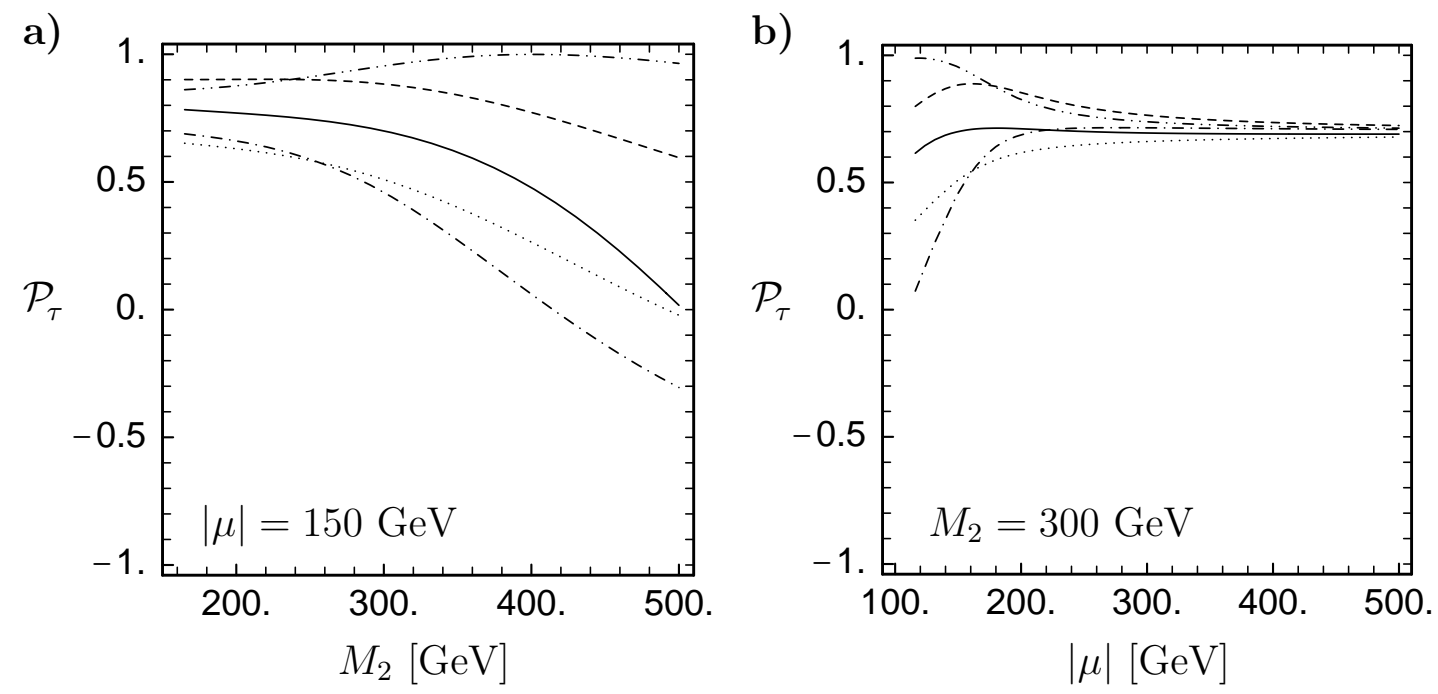

Figure 1: Average polarization of the tau lepton coming from $\tilde{\tau}_{1} \rightarrow \tau \tilde{\chi}_{1}^{0}$ decays for $\theta_{\tilde{\tau}}=130^{\circ}$ and $\tan \beta=10$ : in a) as a function of $M_{2}$ for $|\mu|=150 \mathrm{GeV}$, in b) as a function of $|\mu|$ for $M_{2}=300 \mathrm{GeV}$. The full, dashed, dotted, dash-dotted, and dash-dot-dotted lines are for $\left(\phi_{1}, \varphi_{\tau}\right)=(0,0),\left(0, \frac{\pi}{2}\right),\left(\frac{\pi}{2}, 0\right),\left(\frac{\pi}{2}, \frac{\pi}{2}\right)$, and $\left(\frac{\pi}{2},-\frac{\pi}{2}\right)$, respectively. $M_{2}$ and $\mu$ are taken to be real; for $\left|M_{1}\right|$ the GUT relation $\left|M_{1}\right|=\frac{5}{3} \tan ^{2} \theta_{W} M_{2}$ has been used.

a)

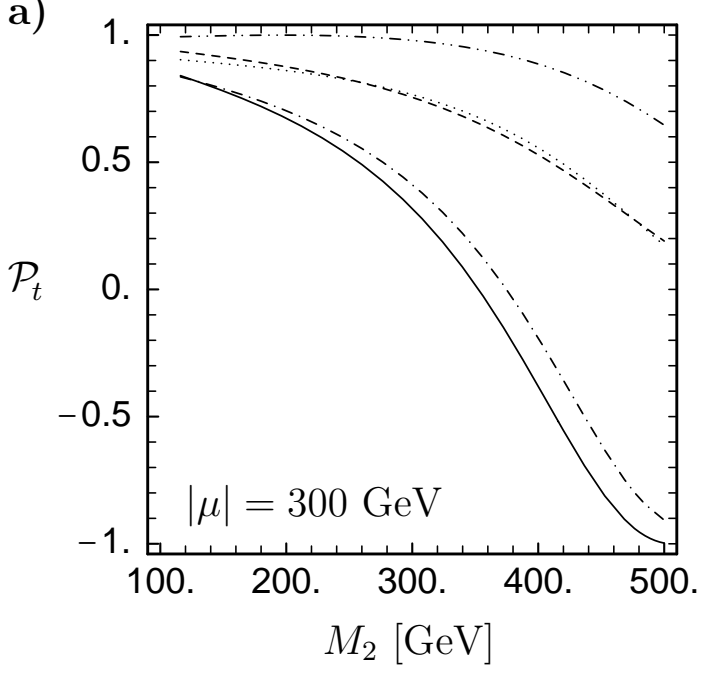

b)

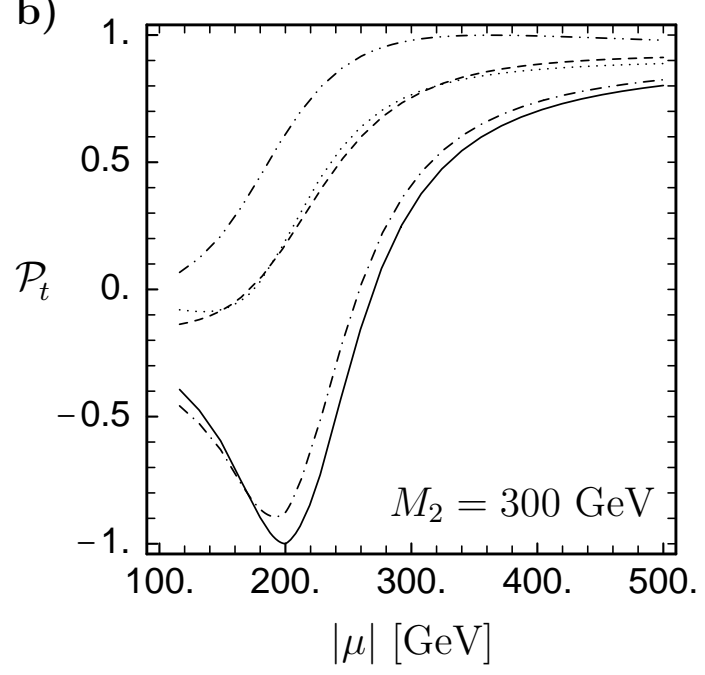

Figure 2: Average polarization of the top quark coming from $\tilde{t}_{1} \rightarrow t \tilde{\chi}_{1}^{0}$ decays analogous to Fig. 1. It is assumed that $m_{\tilde{t}_{1}} \gg m_{t}$. 
vanishing phases as reference point and assume that the following precisions can be achieved: ${ }^{1}$ $\delta M_{1}=\delta M_{2}=\delta \mu=0.5 \%, \delta \tan \beta=1, \delta \theta_{\tilde{\tau}}=3.4^{\circ}$, and $\delta \phi_{1}=\delta \phi_{\mu}=0.1$. Varying the parameters within this range around the reference point leads to $\mathcal{P}_{\tau}=0.39_{-0.27}^{+0.19}$ at $\varphi_{\tilde{\tau}}=0$, which is indicated as an error bar in Fig. 3b. (The 3-5\% uncertainty in the measurement of $\mathcal{P}_{\tau}$ is comparatively negligible). We conclude that in our particular scenario, if no phase has been observed in the neutralino/chargino sector, a measurement of $\mathcal{P}_{\tau}$ would be sensitive to $\left|\varphi_{\tilde{\tau}}\right| \gtrsim 0.3 \pi$. If $\tan \beta$ can be measured to $\tan \beta=10 \pm 0.1$, this improves to $\delta \mathcal{P}_{\tau} \simeq 0.1$ and $\left|\varphi_{\tilde{\tau}}\right| \gtrsim 0.2 \pi$. According to (27), a measurement of a non-zero $\varphi_{\tilde{\tau}}+\phi_{\mu}$ implies a lower limit on $\left|A_{\tau}\right|$; in our example where $\phi_{\mu}=0,\left|A_{\tau}\right|>735 \mathrm{GeV}(1 \mathrm{TeV})$ for $\left|\varphi_{\tilde{\tau}}\right|>0.2 \pi(0.3 \pi)$. Increasing the precision in $\delta M_{1}, \delta M_{1}$ and $\delta|\mu|$ from $0.5 \%$ to $0.1 \%$ barely improves these limits.

We perform a similar analysis for $\mathcal{P}_{t}$, using $M_{2}=225 \mathrm{GeV}$ and $\mu=200 \mathrm{GeV}$ as reference point. The results are shown in Figs. 4a,b in analogy to Figs. 3a,b. Again a high sensitivity to both $\phi_{1}$ and $\varphi_{\tilde{t}}$ is observed. For the case of vanishing phases, we get $\mathcal{P}_{t}=-0.48$. A variation of the parameters around the reference point as above (with $\delta \theta_{\tilde{t}}=3.5^{\circ}$ ) leads to a parametric uncertainty of $\delta \mathcal{P}_{t}^{\text {par }} \simeq 0.2$. Adding the experimental resolution $\delta \mathcal{P}_{t}^{\text {exp }} \simeq 0.1$ in quadrature gives $\mathcal{P}_{t}=-0.48 \pm 0.22$ at $\varphi_{\tilde{t}}=0$, indicated as an error bar in Fig. $4 \mathrm{~b}$. We see that in this scenario $\mathcal{P}_{t}$ would be sensitive to $\left|\varphi_{\tilde{t}}\right| \gtrsim 0.15 \pi$. If $\theta_{\tilde{t}}$ can be measured to $\sim 1^{\circ}$ this improves to $\delta \mathcal{P}_{t}^{\text {par }} \simeq 0.1\left(\delta \mathcal{P}_{t} \simeq 0.14\right)$ and $\left|\varphi_{\tilde{t}}\right| \gtrsim 0.11 \pi$; if $M_{1}, M_{2},|\mu|$ can be measured to $0.1 \%$ and $\tan \beta$ to $0.1, \delta \mathcal{P}_{t}^{\text {par }}$ becomes negligible with respect to the experimental resolution of $\mathcal{P}_{t}$. Since $\varphi_{\tilde{t}} \simeq \phi_{A_{t}}$, a measurement of $\mathcal{P}_{t}$ can be used to derive information on $A_{t}$. In particular, if both mass eigenstates are known, $A_{t}$ is given by

$$
A_{t}=\frac{1}{2 m_{t}}\left(m_{\tilde{t}_{2}}^{2}-m_{\tilde{t}_{1}}^{2}\right)\left|\sin 2 \theta_{\tilde{t}}\right| e^{i \varphi_{\tilde{t}}}+\mu^{*} \cot \beta
$$

An analogous relation with $\cot \beta \rightarrow \tan \beta$ holds for $A_{\tau}$, although the precision on $A_{\tau}$ is in general much worse than on $A_{t}$. In this context note that $\mathcal{P}_{f}$ can also be useful to resolve the sign ambiguity in the $\cos \theta_{\tilde{f}}$ determination from cross section measurements [51] in the CP-conserving case. This corresponds to distinguishing the cases $\varphi_{\tilde{f}}=0$ and $\varphi_{\tilde{f}}=\pi$.

Even though we have presented results of our analysis for $\phi_{\mu}$, chosen in order to satisfy the EDM constraints without having to appeal to cancellations, we have also investigated the case of a non-zero $\phi_{\mu}$. We found that a non-zero $\phi_{\mu}$ shifts the curves in Figs. 1-4 but does not cause a qualitative change of the results.

Last but not least we note that giving up the GUT relation between $\left|M_{1}\right|$ and $M_{2}$ changes the picture completely, as the pattern of gaugino-higgsino mixing is strongly affected [52]. This is illustrated in Fig. 5, where we plot $\mathcal{P}_{\tau}$ and $\mathcal{P}_{t}$ as functions of $r=\left|M_{1}\right| / M_{2}$ for $M_{2}=300 \mathrm{GeV}$, $|\mu|=150 \mathrm{GeV}$ and the other parameters as in Figs. 1 and 2. A detailed study of the implications of non-universal gaugino masses will be presented elsewhere.

To sum up, both $\mathcal{P}_{t}$ and $\mathcal{P}_{\tau}$ can vary over a large range depending on $\phi_{1}$ and $\varphi_{\tilde{t}, \tilde{\tau}}$ (and also $\phi_{\mu}$, though we did not discuss this case explicitly) and may thus be used as sensitive probes of these phases. To this aim, however, the neutralino mass parameters, $\tan \beta$ and the sfermion mixing angles need to be known. Given the complexity of the problem, a combined fit of all available data seems to be the most convenient method for the extraction of the MSSM parameters.

\footnotetext{
${ }^{1}$ We make a somewhat conservative estimate because a full simulation of such a scenario is not available.
} 

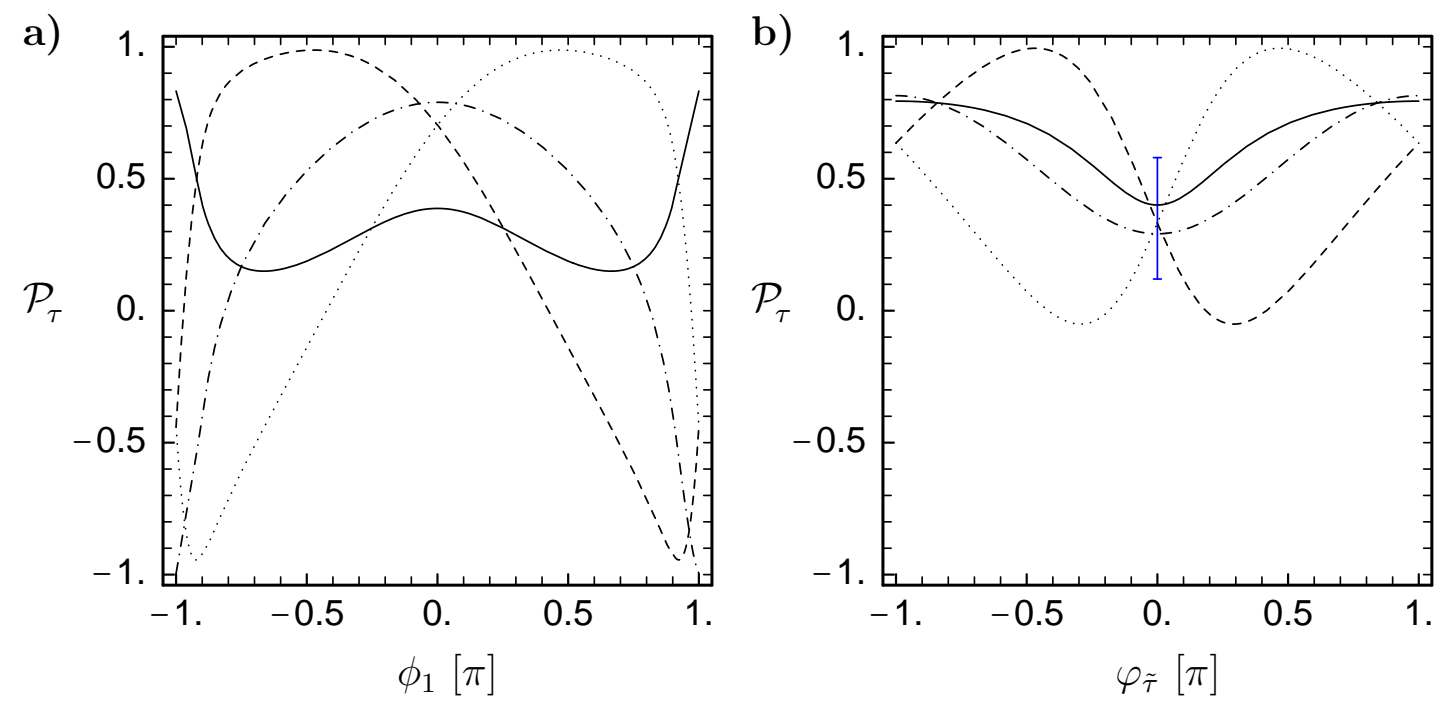

Figure 3: Average polarization of the tau lepton coming from $\tilde{\tau}_{1} \rightarrow \tau \tilde{\chi}_{1}^{0}$ decays for $\theta_{\tilde{\tau}}=130^{\circ}$ and $\tan \beta=10$ : in a) as a function of $\phi_{1}$ for $M_{2}=380 \mathrm{GeV}$ and $|\mu|=125 \mathrm{GeV}$; in b) as a function of $\varphi_{\tilde{\tau}}$ for $|\mu|=125 \mathrm{GeV}$ and $M_{2}$ adjusted such that $m_{\tilde{\chi}_{1}^{0}}=100 \mathrm{GeV}$. The full, dashed, dotted, and dash-dotted lines are for $\varphi_{\tilde{\tau}}\left(\phi_{1}\right)=0, \frac{\pi}{2},-\frac{\pi}{2}, \pi$ in a (b). The error on $\mathcal{P}_{\tau}$ indicated by the vertical bar in b) has been estimated as described in the text.
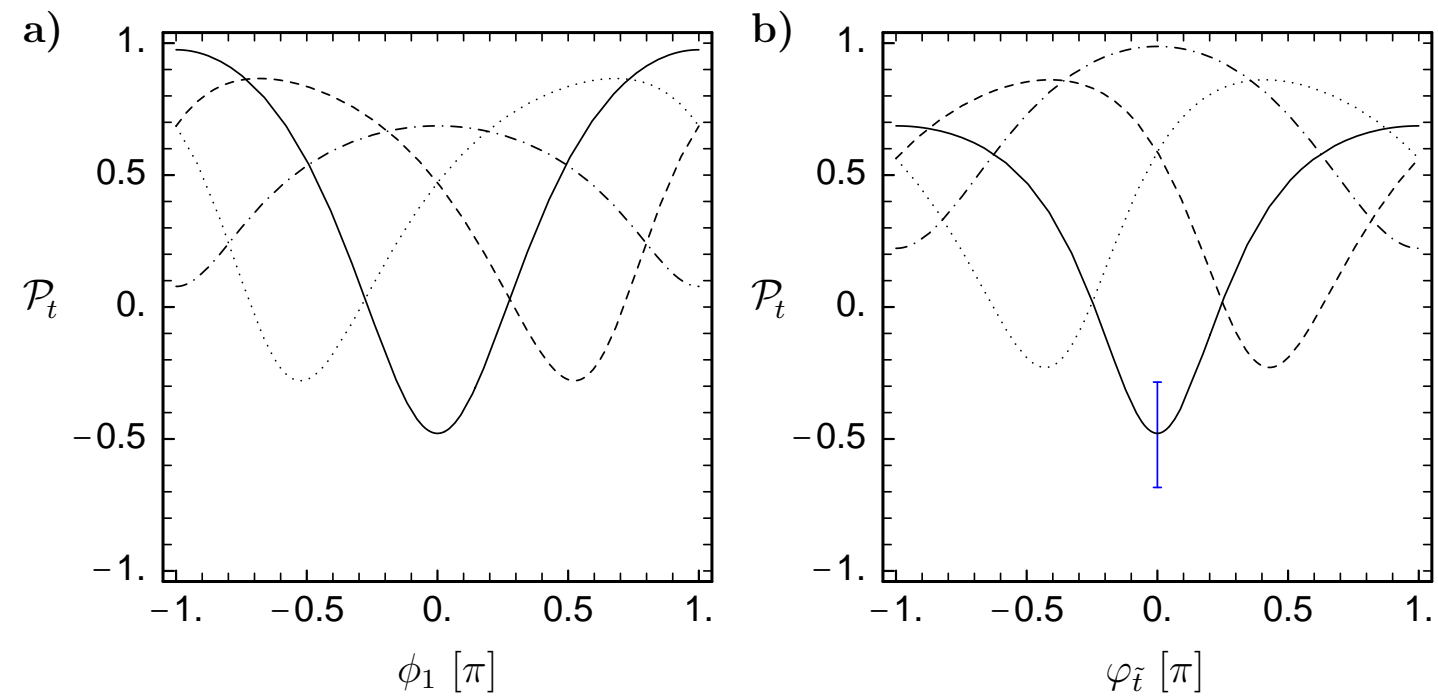

Figure 4: Average polarization of the top quark coming from $\tilde{t}_{1} \rightarrow t \tilde{\chi}_{1}^{0}$ decays for $\theta_{\tilde{t}}=130^{\circ}$, and $\tan \beta=10$ : in a) as a function of $\phi_{1}$ for $M_{2}=225 \mathrm{GeV}$ and $|\mu|=200 \mathrm{GeV}$; in b) as a function of $\varphi_{\tilde{t}}$ for $|\mu|=200 \mathrm{GeV}$ and $M_{2}$ adjusted such that $m_{\tilde{\chi}_{1}^{0}}=100 \mathrm{GeV}$. The full, dashed, dotted, and dash-dotted lines are for $\varphi_{\tilde{t}}\left(\phi_{1}\right)=0, \frac{\pi}{2},-\frac{\pi}{2}, \pi$ in a (b). The error on $\mathcal{P}_{t}$ indicated by the vertical bar in b) has been estimated as described in the text. 

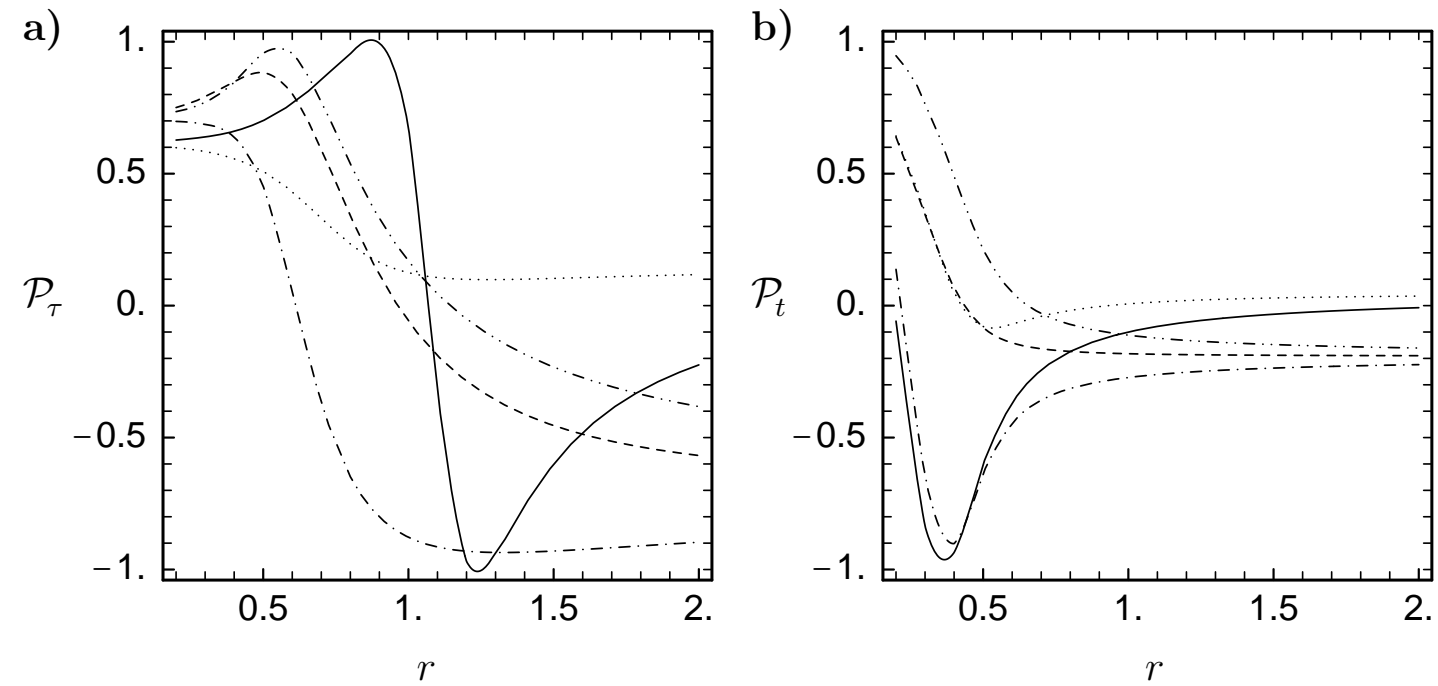

Figure 5: $\mathcal{P}_{\tau}$ (left) and $\mathcal{P}_{t}$ (right) as functions of $r=\left|M_{1}\right| / M_{2}$, for $M_{2}=300 \mathrm{GeV}$ and $|\mu|=$ $150 \mathrm{GeV}$. The full, dashed, dotted, dash-dotted, and dash-dot-dotted lines are for $\left(\phi_{1}, \varphi_{\tilde{\tau}, \tilde{t}}\right)=$ $(0,0),\left(0, \frac{\pi}{2}\right),\left(\frac{\pi}{2}, 0\right),\left(\frac{\pi}{2}, \frac{\pi}{2}\right)$ and $\left(\frac{\pi}{2},-\frac{\pi}{2}\right)$, respectively.

\section{Fermion polarization in $\tilde{f} \rightarrow f^{\prime} \tilde{\chi}^{ \pm}$decays}

The sfermion interaction with charginos is $(i, j=1,2)$

$$
\mathcal{L}_{f^{\prime} \tilde{f} \tilde{\chi}^{ \pm}}=g \bar{u}\left(l_{i j}^{\tilde{d}} P_{R}+k_{i j}^{\tilde{d}} P_{L}\right) \tilde{\chi}_{j}^{+} \tilde{d}_{i}+g \bar{d}\left(l_{i j}^{\tilde{u}} P_{R}+k_{i j}^{\tilde{u}} P_{L}\right) \tilde{\chi}_{j}^{+c} \tilde{u}_{i}+\text { h.c. }
$$

where $u(\tilde{u})$ stands for up-type (s)quark and (s)neutrinos, and $d(\tilde{d})$ stands for down-type (s)quark and charged (s)leptons. The couplings $l$ and $k$ are

$$
\begin{aligned}
l_{i j}^{\tilde{t}} & =-V_{j 1} R_{i 1}^{\tilde{t} *}+h_{t} V_{j 2} R_{i 2}^{\tilde{t} *}, & l_{i j}^{\tilde{b}} & =-U_{j 1} R_{i 1}^{\tilde{b} *}+h_{b} U_{j 2} R_{i 2}^{\tilde{b} *}, \\
k_{i j}^{\tilde{t}} & =h_{b} U_{j 2}^{*} R_{i 1}^{\tilde{t} *}, & k_{i j}^{\tilde{b}} & =h_{t} V_{j 2}^{*} R_{i 1}^{\tilde{b} *},
\end{aligned}
$$

for stops and sbottoms and

$$
\begin{aligned}
l_{j}^{\tilde{\nu}} & =-V_{j 1}, & l_{i j}^{\tilde{\tau}} & =-U_{j 1} R_{i 1}^{\tilde{\tau} *}+h_{\tau} U_{j 2} R_{i 2}^{\tilde{\tau} *}, \\
k_{j}^{\tilde{\nu}} & =h_{\tau} U_{j 2}^{*}, & k_{i j}^{\tilde{\tau}} & =0
\end{aligned}
$$

for staus and sneutrinos.

Analogous to the decay into a neutralino, eq. (23), the average polarization of the fermion coming from the $\tilde{f}_{i} \rightarrow f^{\prime} \tilde{\chi}_{j}^{ \pm}$decay is given by

$$
\mathcal{P}_{f}^{\prime}=\frac{\operatorname{Br}\left(\tilde{f}_{i} \rightarrow \tilde{\chi}_{j}^{ \pm} f_{R}^{\prime}\right)-\operatorname{Br}\left(\tilde{f}_{i} \rightarrow \tilde{\chi}_{j}^{ \pm} f_{L}^{\prime}\right)}{\operatorname{Br}\left(\tilde{f}_{i} \rightarrow \tilde{\chi}_{j}^{ \pm} f_{R}^{\prime}\right)+\operatorname{Br}\left(\tilde{f}_{i} \rightarrow \tilde{\chi}_{j}^{ \pm} f_{L}^{\prime}\right)}=\frac{\left|k_{i j}^{\tilde{f}}\right|^{2}-\left|l_{i j}^{\tilde{f}}\right|^{2}}{\left|k_{i j}^{\tilde{f}}\right|^{2}+\left|l_{i j}^{\tilde{f}}\right|^{2}} .
$$

Since only top and tau polarizations are measurable, we only discuss $\tilde{b} \rightarrow t \tilde{\chi}^{-}$and $\tilde{\nu}_{\tau} \rightarrow \tau \tilde{\chi}^{+}$ decays. The latter case is especially simple because $\mathcal{P}_{\tau}^{\prime}$ depends only on the parameters of the chargino sector:

$$
\left(\mathcal{P}_{\tau}^{\prime}\right)_{j}=\mathcal{P}_{\tau}^{\prime}\left(\tilde{\nu}_{\tau} \rightarrow \tau \tilde{\chi}_{j}^{+}\right)=\frac{\left|h_{\tau} U_{j 2}\right|^{2}-\left|V_{j 1}\right|^{2}}{\left|h_{\tau} U_{j 2}\right|^{2}+\left|V_{j 1}\right|^{2}} .
$$




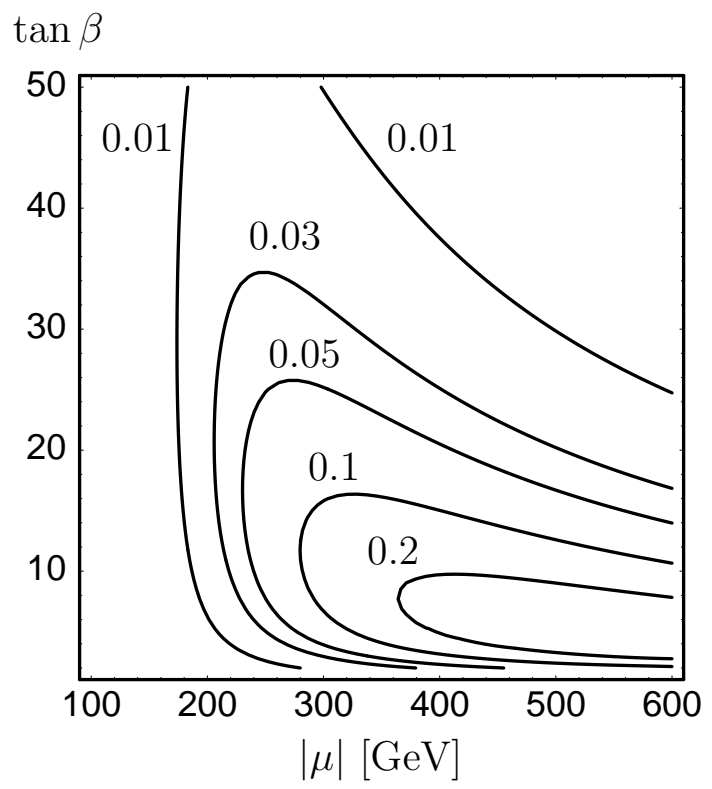

Figure 6: Contours of constant $\Delta\left(\mathcal{P}_{\tau}^{\prime}\right)_{2}=\mathcal{P}_{\tau}^{\prime}\left(\tilde{\nu}_{\tau} \rightarrow \tau \tilde{\chi}_{2}^{+}, \phi_{\mu}=\frac{\pi}{2}\right)-\mathcal{P}_{\tau}^{\prime}\left(\tilde{\nu}_{\tau} \rightarrow \tau \tilde{\chi}_{2}^{+}, \phi_{\mu}=0\right)$ in the $(\tan \beta,|\mu|)$ plane for $M_{2}=150 \mathrm{GeV}$.

A measurement of $\mathcal{P}_{\tau}{ }^{\prime}$ may hence be useful to supplement the chargino parameter determination. However, the dependence of $\mathcal{P}_{\tau}^{\prime}\left(\tilde{\nu}_{\tau} \rightarrow \tau \tilde{\chi}_{1}^{+}\right)$on $\phi_{\mu}$ turns out to be very small, the effects being in general well below $1 \%$ (i.e. $\Delta \mathcal{P}<0.01$ ). Only for the decay into the heavier chargino, the effect of a non-zero phase ${ }^{2}$ may be sizeable. As an example, Fig. 6 shows the differences in $\left(\mathcal{P}_{\tau}^{\prime}\right)_{2}$ between $\phi_{\mu}=0$ and $\phi_{\mu}=\pi / 2$ in the $(\tan \beta,|\mu|)$ plane for $M_{2}=150 \mathrm{GeV}$. $\Delta\left(\mathcal{P}_{\tau}^{\prime}\right)_{2}=\mathcal{P}_{\tau}^{\prime}\left(\tilde{\nu}_{\tau} \rightarrow \tau \tilde{\chi}_{2}^{+}, \phi_{\mu}=\frac{\pi}{2}\right)-\mathcal{P}_{\tau}^{\prime}\left(\tilde{\nu}_{\tau} \rightarrow \tau \tilde{\chi}_{2}^{+}, \phi_{\mu}=0\right)$ can go up to $\sim 0.25$. However, it requires quite heavy sneutrinos for this decay to be kinematically allowed. Moreover, the measurement of $\left(\mathcal{P}_{\tau}^{\prime}\right)_{2}$ will be diluted by $\tilde{\nu}_{\tau} \rightarrow \tau \tilde{\chi}_{1}^{+}$decays.

Let us now turn to top polarization in $\tilde{b} \rightarrow t \tilde{\chi}^{-}$decays. For $\tilde{b}_{1}$ decays, we have

$$
\begin{aligned}
\left|k_{1 j}^{\tilde{b}}\right|^{2}-\left|l_{1 j}^{\tilde{b}}\right|^{2}= & h_{t}^{2} \cos ^{2} \theta\left|V_{j 2}^{*} e^{-i \varphi}\right|^{2}-\left|h_{b} U_{j 2} \sin \theta-U_{j 1} \cos \theta e^{-i \varphi}\right|^{2} \\
= & \left(h_{t}^{2}\left|V_{j 2}\right|^{2}-\left|U_{j 1}\right|^{2}\right) \cos ^{2} \theta-h_{b}^{2}\left|U_{j 2}\right|^{2} \sin ^{2} \theta \\
& +h_{b} \sin 2 \theta\left(\mathcal{R} e\left[U_{j 1} U_{j 2}^{*}\right] \cos \varphi+\mathcal{I} m\left[U_{j 1} U_{j 2}^{*}\right] \sin \varphi\right) .
\end{aligned}
$$

For $\tilde{b}_{2}$ decays, the corresponding expression is given by the RHS of (36) with $\cos ^{2} \theta, \sin ^{2} \theta$ interchanged, and a change in sign of the term $\propto \sin 2 \theta$. We see that the phase dependence of $\mathcal{P}_{t}^{\prime}$ is proportional to $h_{b} \sin 2 \theta_{\tilde{b}}$ and the amount of gaugino-higgsino mixing of the charginos; it will therefore be largest for $\left|M_{2}\right| \sim|\mu|, \theta_{\tilde{b}}=3 \pi / 4$ and large $\tan \beta$. Again, there is a non-zero effect even if there is just one phase in either the sbottom or chargino sector. Note, however, that the only $\mathrm{CP}$ phase in the chargino sector is $\phi_{\mu}$, which also enters the sfermion mass matrices. Complex $U$ and $V$ hence imply $\varphi_{\tilde{b}} \neq 0$. More precisely, $a_{b}=\left|a_{b}\right| e^{i \varphi_{\tilde{b}}} \sim \mu^{*} \tan \beta=|\mu| \tan \beta e^{-i \phi_{\mu}}$ for medium and large $\tan \beta$, and thus $\varphi_{\tilde{b}}=-\phi_{\mu}$ unless $\left|A_{b}\right| \gtrsim|\mu| \tan \beta$; see eq. (27) and the related discussion. For the sake of a general discussion of the phase dependence of $\mathcal{P}_{t}^{\prime}$ (and since $A_{b}$ is still a free parameter), we nevertheless use $\phi_{\mu}$ and $\varphi_{\tilde{b}}$ as independent input parameters.

\footnotetext{
${ }^{2}$ We remind the reader that unless huge cancellations are invoked, $\phi_{\mu}$ is severely restricted by the nonobservation of the eEDM.
} 
Figure 7 shows the average top polarization in $\tilde{b}_{1} \rightarrow t \tilde{\chi}_{1}^{-}$decays as a function of $|\mu|$ for $M_{2}=225 \mathrm{GeV}, \tan \beta=10$ and 30 , and various combinations of $\phi_{\mu}$ and $\varphi_{\tilde{b}}$. Here we have fixed $\theta_{\tilde{b}}=140^{\circ}$, since from renormalization-group running one expects $m_{\tilde{b}_{L}}<m_{\tilde{b}_{R}}$. As in the previous section, we find large effects from $\mathrm{CP}$-violating phases if the $\tilde{\chi}^{ \pm}$has a sizeable higgsino component; as expected, these effects are enhanced for large $\tan \beta$. The results stay the same if both $\phi_{\mu}$ and $\varphi_{\tilde{b}}$ change their signs. Moreover, $\mathcal{P}_{t}^{\prime}\left(\phi_{\mu}=0, \varphi_{\tilde{b}}=\frac{\pi}{2}\right) \sim \mathcal{P}_{t}^{\prime}\left(\phi_{\mu}=\frac{\pi}{2}, \varphi_{\tilde{b}}=0\right)$. If $\phi_{\mu}$ and $\varphi_{\tilde{b}}$ have the same sign, the difference in $\mathcal{P}_{t}^{\prime}$ from the case of vanishing phases is larger than if they have opposite signs. In particular, we find $\mathcal{P}_{t}^{\prime}\left(\phi_{\mu}=-\varphi_{\tilde{b}}\right) \sim \mathcal{P}_{t}^{\prime}\left(\phi_{\mu}=\varphi_{\tilde{b}}=0\right)$ over large regions of the parameter space. With an experimental resolution of the top polarization of about $10 \%$ this implies that in many cases $\varphi_{\tilde{b}} \sim-\phi_{\mu}$ cannot be distinguished from $\varphi_{\tilde{b}}=\phi_{\mu}=0$ by measurement of $\mathcal{P}_{t}^{\prime}$. Furthermore, the value of $\mathcal{P}_{t}^{\prime}$ is quite sensitive to the running $b$ quark mass, which enters the bottom Yukawa coupling of eq. (22) and is subject to possibly large SUSY loop corrections. For the lines in Fig. 7 we have used $m_{b}=4.5 \mathrm{GeV}$. The grey bands show the range of $\mathcal{P}_{t}^{\prime}$ when $m_{b}$ is varied between 2.5 and $4.5 \mathrm{GeV}$. As can be seen, the uncertainty in $m_{b}$ - more precisely in $h_{b}$ - tends to wash out small effects of CP-violating phases, specially in the case of large $\tan \beta$.

In order to see what information can be extracted from a $\mathcal{P}_{t}^{\prime}$ measurement, we pick two values of $|\mu|$ from Fig. 7a; namely $|\mu|=200 \mathrm{GeV}$ and $|\mu|=350 \mathrm{GeV}$, and show the phase dependences at these points in Fig. 8. Figure 8 a shows $\mathcal{P}_{t}^{\prime}$ as a function of $\varphi_{\tilde{b}}$, for $|\mu|=200 \mathrm{GeV}, \tan \beta=10$, $\theta_{\tilde{b}}=140^{\circ}$, and various values of $\phi_{\mu} . M_{2}$ is chosen such that $m_{\tilde{\chi}_{1}^{ \pm}}=155 \mathrm{GeV}$ (i.e. $M_{2}=225 \mathrm{GeV}$ for $\left.\phi_{\mu}=0\right)$. The range obtained by varying $m_{b}$ within $2.5-4.5 \mathrm{GeV}$ is shown as grey bands for two of the curves, for $\phi_{\mu}=0$ and $\phi_{\mu}=-\varphi_{\tilde{b}}$. We estimate the effect of an imperfect knowledge of the model parameters in the same way as in the previous section. For $M_{2}=225 \pm 1.125 \mathrm{GeV}$, $|\mu|=200 \pm 1 \mathrm{GeV}, \tan \beta=10 \pm 1, \theta_{\tilde{b}}=140 \pm 3.4^{\circ}$ and $\phi_{\mu}=0 \pm 0.1$, we get $\mathcal{P}_{t}^{\prime}=0.89 \pm 0.06$ at $\varphi_{\tilde{b}}=0$. Varying in addition $m_{b}=2.5-4.5 \mathrm{GeV}$ gives $\mathcal{P}_{t}^{\prime}=0.89_{-0.16}^{+0.06}$. Adding a $10 \%$ measurement error on $\mathcal{P}_{t}^{\prime}$ in quadrature, we end up with $\delta \mathcal{P}_{t}^{\prime}=0.12(0.19)$ without (with) the $m_{b}$ effect. These are shown as error bars in Fig. 8a. We see that the case of $\varphi_{\tilde{b}}=-\phi_{\mu}$ cannot be distinguished from $\varphi_{\tilde{b}}=\phi_{\mu}=0$ in this scenario. However, $\mathcal{P}_{t}^{\prime}$ turns out to be quite a sensitive probe of $\delta_{\phi}=\varphi_{\tilde{b}}+\phi_{\mu}$, i.e. the deviation from the 'natural' alignment $\varphi_{\tilde{b}}=-\phi_{\mu}$. In the example of Fig. 8a, $\left|\delta_{\phi}\right| \gtrsim 0.24 \pi(0.31 \pi)$ can be resolved if $h_{b}$ is (not) known precisely, quite independently of $\phi_{\mu}$. Observing such a $\delta_{\phi}$ also implies a bound on $\left|A_{b}\right|$ of $\left|A_{b}\right|>1363$ (1678) $\mathrm{GeV}$. If the precision on $M_{2}$ and $|\mu|$ is $0.1 \%$ and $\tan \beta=10 \pm 0.1$, we get $\left(\delta \mathcal{P}_{t}^{\prime}\right)^{\text {par }}=0.03$ at $\varphi_{\tilde{b}}=0$, so that the error is dominated by the experimental uncertainty. However, the resultant improvement in the sensitivity is limited to $\left|\delta_{\phi}\right| \gtrsim 0.22 \pi$ and $\left|A_{b}\right|>1294 \mathrm{GeV}$.

Figure $8 \mathrm{~b}$ shows $\mathcal{P}_{t}^{\prime}$ as a function of $\varphi_{\tilde{b}}$, for $|\mu|=350 \mathrm{GeV}, m_{\tilde{\chi}_{1}^{ \pm}}=204 \mathrm{GeV}$, and the other parameters as above $\left(M_{2}=225 \mathrm{GeV}\right.$ at $\left.\phi_{\mu}=0\right)$. The effect of an uncertainty in $h_{b}$ is again shown as grey bands for $\phi_{\mu}=0$ and $\phi_{\mu}=-\varphi_{\tilde{b}}$. Estimating the parametric uncertainty in the same way as above, but with $|\mu|=350 \pm 1.75 \mathrm{GeV}$, we get $\mathcal{P}_{t}^{\prime}=-0.43_{-0.04}^{+0.06}$ at $\varphi_{\tilde{b}}=0$. Varying in addition $m_{b}=2.5-4.5 \mathrm{GeV}$ gives $\mathcal{P}_{t}^{\prime}=-0.43_{-0.09}^{+0.06}$. Adding a $10 \%$ measurement error on $\mathcal{P}_{t}^{\prime}$ in quadrature, we end up with $\delta \mathcal{P}_{t}^{\prime}=0.11(0.14)$ without (with) the effect of $m_{b}$, shown as error bars in Fig. 8b. In a three-dimensional plot, $\mathcal{P}_{t}^{\prime}$ has a bell-like shape in the $\phi_{\mu}-\varphi_{\tilde{b}}$ plane, with contours of constant $\mathcal{P}_{t}^{\prime}$ being ellipses in this plane. If $\phi_{\mu}$ is not known, a measurement of $\mathcal{P}_{t}^{\prime}$ may therefore be useful to put limits on $\phi_{\mu}$ and $\varphi_{\tilde{b}}$, but not on $\delta_{\phi}$, which restricts $\left|A_{b}\right|$. In our case study, we have assumed that $\phi_{\mu}=0$ is known. In this case, a measurement of $\mathcal{P}_{t}^{\prime}=-0.43$, for instance, would restrict $\left|\varphi_{\tilde{b}}\right| \lesssim 0.38 \pi$ at $1 \sigma$, while a measurement of $\mathcal{P}_{t}^{\prime}=-0.56$ would disfavour $\left|\varphi_{\tilde{b}}\right| \gtrsim 0.9 \pi$ as well as $\left|\varphi_{\tilde{b}}\right| \lesssim 0.13 \pi$. The latter would also allow a constraint on $\delta_{\phi}$. 

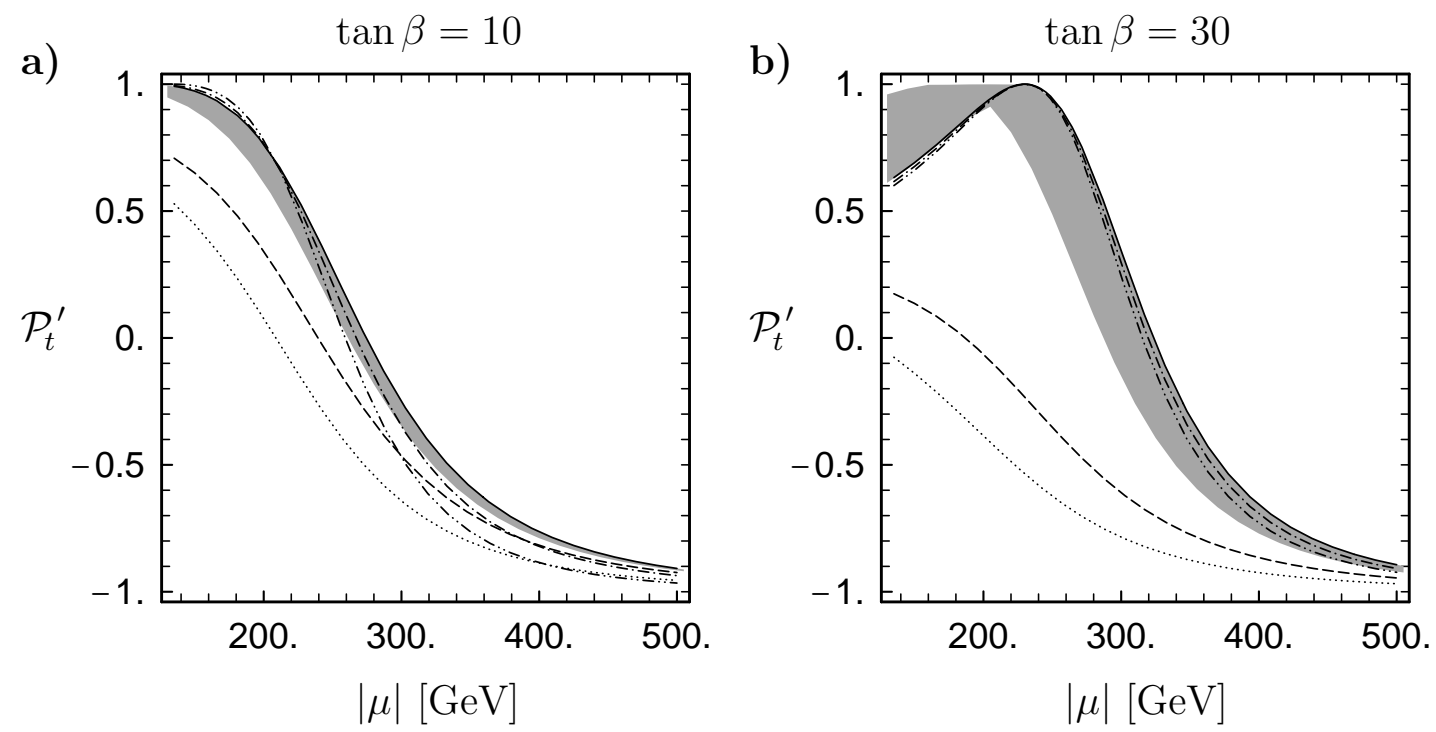

Figure 7: Average polarization of the top quark coming from $\tilde{b}_{1} \rightarrow t \tilde{\chi}_{1}^{-}$decays as a function of $|\mu|$ for $\theta_{\tilde{b}}=140^{\circ}, M_{2}=225 \mathrm{GeV}, \tan \beta=10$ in a) and $\tan \beta=30$ in b). The full, dashed, dotted, dash-dotted and dash-dot-dotted lines are for $\left(\phi_{\mu}, \varphi_{\tilde{b}}\right)=(0,0),\left(0, \frac{\pi}{2}\right),\left(\frac{\pi}{2}, \frac{\pi}{2}\right),\left(-\frac{\pi}{2}, \frac{\pi}{2}\right)$, and $(-\pi, \pi)$ respectively. The grey bands show the range of $\mathcal{P}_{t}^{\prime}$ due to varying $m_{b}$ within $2.5-4.5$ $\mathrm{GeV}$ for the case $\phi_{\mu}=0$.
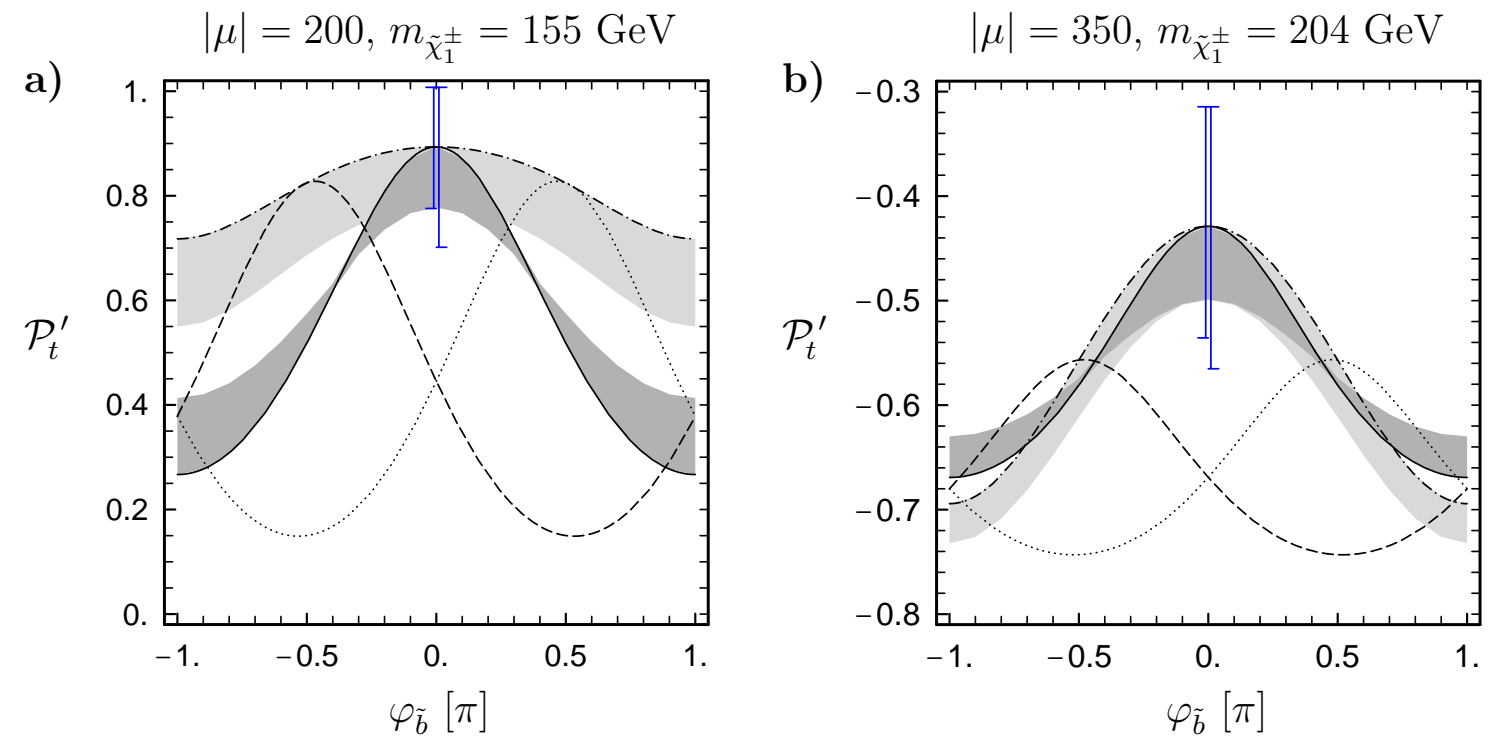

Figure 8: Average polarization of the top quark coming from $\tilde{b}_{1} \rightarrow t \tilde{\chi}_{1}^{-}$decays as a function of $\varphi_{\tilde{b}}$, for $\theta_{\tilde{b}}=140^{\circ}, \tan \beta=10,|\mu|=200$ and $m_{\tilde{\chi}_{1}^{ \pm}}=155 \mathrm{GeV}$ in a) and $|\mu|=350$ and $m_{\tilde{\chi}_{1}^{ \pm}}=204 \mathrm{GeV}$ in b). The full, dashed and dotted lines are for $\phi_{\mu}=0, \frac{\pi}{2}$ and $-\frac{\pi}{2}$, respectively, while for the dash-dotted lines $\phi_{\mu}=-\varphi_{\tilde{b}}$. The grey bands show the range of $\mathcal{P}_{t}^{\prime}$ due to varying $m_{b}$ within $2.5-4.5 \mathrm{GeV}$ for the cases $\phi_{\mu}=0$ and $\phi_{\mu}=-\varphi_{\tilde{b}}$. The error bars show the estimated errors on $\mathcal{P}_{t}^{\prime}$ as described in the text. 
As mentioned above, a lower limit on $\delta_{\phi}$ implies a lower limit on $\left|A_{b}\right|$. An upper limit on $\delta_{\phi}$ can be used to set an upper limit on $\left|A_{b}\right|$ as a function of $\phi_{A_{b}}:\left|A_{b}\right|<\left|\frac{\sin \left(\varphi_{\tilde{b}}+\phi_{\mu}\right)}{\sin \left(\varphi_{\tilde{b}}-\phi_{A_{b}}\right)}\right| \times|\mu| \tan \beta$. Note, however, that this becomes unbounded for $\phi_{A_{b}} \rightarrow \varphi_{\tilde{b}}$.

We have also investigated the case of large $\tan \beta(\tan \beta=30)$. It reveals a $\varphi_{\tilde{b}}$ dependence similar to that of Fig. 8a, with almost no dependence on $\phi_{\mu}$ and the $\delta_{\phi}$ dependence accordingly more pronounced. We encounter, however, a large parametric uncertainty, which practically washes out the sensitivity to $\delta_{\phi}$.

To sum up, tau polarization in $\tilde{\nu}_{\tau} \rightarrow \tau \tilde{\chi}^{+}$decays depends only little on $\phi_{\mu}$. $\mathcal{P}_{\tau}^{\prime}$ is hence not a promising quantity to study CP phases, but may be useful for (consistency) tests of the gaugino-higgsino mixing. Top polarization in $\tilde{b} \rightarrow t \tilde{\chi}^{-}$decays, on the other hand, can be useful to probe $\phi_{\mu}, \varphi_{\tilde{b}}$ and/or $\delta_{\phi}=\phi_{\mu}+\varphi_{\tilde{b}}$ in some regions of the parameter space. The measurement of $\mathcal{P}_{t}^{\prime}$, revealing phases or being consistent with vanishing phases, may also constrain $\left|A_{b}\right|$.

\section{Conclusions}

We have discussed the influence of $\mathrm{CP}$-violating phases on the fermion polarization in sfermion decays to neutralinos or charginos, $\tilde{f}_{i} \rightarrow f \tilde{\chi}_{n}^{0}$ and $\tilde{f}_{i} \rightarrow f^{\prime} \tilde{\chi}_{j}^{ \pm}\left(i, j=1,2 ; n=1, \ldots, 4 ; f, f^{\prime}=\right.$ $t, \tau)$. This polarization is considered as a useful tool for the MSSM parameter determination [46-48].

In decays into charginos, the polarization depends on the phase of $\mu$. Since this dependence is weak in the case of $\tilde{\nu}_{\tau} \rightarrow \tau \tilde{\chi}^{+}, \mathcal{P}_{\tau}^{\prime}$ does not provide a promising probe of CP phases (on the other hand, exactly this feature can make $\mathcal{P}_{\tau}{ }^{\prime}$ useful for consistency tests of gaugino-higgsino mixing). In $\tilde{b} \rightarrow t \chi^{-}$decays, the dependence on $\phi_{\mu}$ can be rather large; in addition, also the phase of the sbottom-mixing matrix plays a role. If $\left|A_{b}\right|<|\mu| \tan \beta, \varphi_{\tilde{b}} \simeq-\phi_{\mu}$. We found that this case can be difficult to distinguish from the CP-conserving case by measuring $\mathcal{P}_{t}^{\prime}$. If, however, a deviation from $\varphi_{\tilde{b}}+\phi_{\mu}=0$ is observed, these phases can be constrained and also limits on $A_{b}$ can be derived.

The decays $\tilde{t} \rightarrow t \tilde{\chi}^{0}$ and $\tilde{\tau} \rightarrow \tau \tilde{\chi}^{0}$ provide a more effective probe of CP violation because an additional phase, the phase of $M_{1}$, contributes. We found that CP phases can have a significant effect on the top and tau polarizations, especially if the involved neutralino has a sizeable higgsino component. If the parameters of the neutralino sector can be measured precisely, e.g. in $e^{+} e^{-}$annihilation with polarized beams, $\mathcal{P}_{t}$ and $\mathcal{P}_{\tau}$ can be useful for the determination of $\mathrm{CP}$ phases. In particular, since $\varphi_{\tilde{t}} \simeq \phi_{A_{t}}$ unless $|\mu|$ is very large, a measurement of $\mathcal{P}_{t}$ can give information on $A_{t}$.

In this respect it is important to note that (for fixed masses) the sfermion production cross sections do not depend on CP phases. In the sfermion sector, these can be manifest in branching ratios as discussed in $[35,36,38,39]$, polarization of the decay fermions as discussed in this paper, and CP-odd asymmetries. Branching ratios are in general rather difficult to measure with high precision. The information that can be gained from branching ratios is also limited if one decay channel dominates, e.g. $\tilde{\tau}_{1} \rightarrow \tau \tilde{\chi}_{1}^{0}$ in case of a light stau. This makes the polarization of the decay fermions a very interesting possibility to explore CP phases. Last but not least we note that the computations in this paper, leading to effects of a few percent, have been performed at tree level. The influence of radiative corrections [53] can be of comparable size and will therefore have to be taken into account for precision analyses. 
A measurement of the $\mathrm{CP}$ phases in the sfermion $/ \tilde{\chi}^{ \pm}, \tilde{\chi}^{0}$ sector will also complement $\mathrm{CP}$ studies of the Higgs sector [54], since in the MSSM Higgs-sector CP violation is generated through quantum corrections [13-16]. Last but not least we emphazise that, since the effects can be large, the possibility of CP violation should be taken into account in precision SUSY parameter analyses, especially in a general analysis project as envisaged in [55].

\section{Acknowledgements}

T.G. acknowledges the financial support of the Institut für Hochenergiephysik in Vienna and the CERN Theory Division. R.G. wishes to thank the CERN Theory Division and LAPTH for hospitality and financial support, where this work was completed. S.K. thanks the Centre for High Energy Physics in Bangalore for hospitality and financial support. RG wishes to acknowledge the partial support of the Department of Science and Technology, India, under project number SP/S2/K-01/2000-II.

\section{A Standard Model constants}

The SM constants used in the numerical analysis are:

$$
\begin{aligned}
m_{t} & =175 \mathrm{GeV} \\
m_{b} & =4.5 \mathrm{GeV} \\
m_{\tau} & =1.77 \mathrm{GeV} \\
m_{Z} & =91.2 \mathrm{GeV} \\
m_{W} & =80.03 \mathrm{GeV} \\
\sin ^{2} \theta_{W} & =0.23 \\
\alpha\left(m_{Z}\right) & =1 / 129
\end{aligned}
$$

\section{References}

[1] J.H. Christenson, J.W. Cronin, V.L. Fitch and R. Turlay, Phys. Rev. Lett. 13, 138 (1964).

[2] BELLE Collab., A. Abashian et al., Phys. Rev. Lett. 86, 2509 (2001), hep-ex/0102018; BABAR Collab., B. Aubert et al., Phys. Rev. Lett. 86, 2515 (2001), hep-ex/0102030.

[3] A. D. Sakharov, Pisma Zh. Eksp. Teor. Fiz. 5 (1967) 32, JETP Lett. 6, 24 (1967).

[4] For a recent summary, see A. D. Dolgov, hep-ph/0211260.

[5] C. L. Bennett et al., Astrophys. J. Suppl. 148, 1 (2003) [astro-ph/0302207].

[6] For a review, see e.g. M. Dine and A. Kusenko, Rev. Mod. Phys. 76, 1 (2004) [hep$\mathrm{ph} / 0303065]$.

[7] See, e.g. H. E. Haber and G. L. Kane, Phys. Rept. 117 (1985) 75. 
[8] T. Ibrahim and P. Nath, Phys. Lett. B 418, 98 (1998) [hep-ph/9707409]; Phys. Rev. D 57, 478 (1998), Erratum-ibid. D 58, 019901 (1998) [hep-ph/9708456]; Phys. Rev. D 58, 111301 (1998), Erratum-ibid. D 60, 099902 (1999) [hep-ph/9807501]; Phys. Rev. D 61, 093004 (2000) [hep-ph/9910553].

[9] M. Brhlik, G. J. Good and G. L. Kane, Phys. Rev. D 59, 115004 (1999) [hep-ph/9810457].

[10] A. Bartl, T. Gajdosik, W. Porod, P. Stockinger and H. Stremnitzer, Phys. Rev. D 60, 073003 (1999) [hep-ph/9903402].

[11] T. Falk and K. A. Olive, Phys. Lett. B 439, 71 (1998) [hep-ph/9806236].

[12] T. Falk, K. A. Olive, M. Pospelov and R. Roiban, Nucl. Phys. B 560, 3 (1999) [hepph/9904393].

[13] A. Pilaftsis, Phys. Rev. D 58 (1998) 096010 [hep-ph/9803297]; Phys. Lett. B 435 (1998) 88 [hep-ph/9805373]; A. Pilaftsis and C. E. Wagner, Nucl. Phys. B 553, 3 (1999) [hepph/9902371].

[14] D. A. Demir, Phys. Rev. D 60 (1999) 055006 [hep-ph/9901389].

[15] S. Y. Choi, M. Drees and J. S. Lee, Phys. Lett. B 481, 57 (2000) [hep-ph/0002287].

[16] M. Carena, J. R. Ellis, A. Pilaftsis and C. E. M. Wagner, Nucl. Phys. B 586, 92 (2000) [hep-ph/0003180].

[17] D. Chang, W. Y. Keung and A. Pilaftsis, Phys. Rev. Lett. 82, 900 (1999) [Erratum-ibid. 83, 3972 (1999)] [arXiv:hep-ph/9811202].

[18] A. Pilaftsis, Phys. Lett. B 471, 174 (1999) [arXiv:hep-ph/9909485].

[19] D. A. Demir, O. Lebedev, K. A. Olive, M. Pospelov and A. Ritz, Nucl. Phys. B 680 (2004) 339 [arXiv:hep-ph/0311314].

[20] A. Dedes and S. Moretti, Phys. Rev. Lett. 84 (2000) 22, [hep-ph/9908516]; Nucl. Phys. B 576 (2000) 29, [hep-ph/990941].

[21] S. Y. Choi, K. Hagiwara and J. S. Lee, Phys. Lett. B 529 (2002) 212 [hep-ph/0110138].

[22] S. Y. Choi, A. Djouadi, H. S. Song and P. M. Zerwas, Eur. Phys. J. C 8, 669 (1999) [hep-ph/9812236].

[23] S. Y. Choi, M. Guchait, H. S. Song and W. Y. Song, Phys. Lett. B 483, 168 (2000) [hep-ph/9904276].

[24] J. L. Kneur and G. Moultaka, Phys. Rev. D 61, 095003 (2000) [hep-ph/9907360].

[25] S. Y. Choi, A. Djouadi, M. Guchait, J. Kalinowski, H. S. Song and P. M. Zerwas, Eur. Phys. J. C 14, 535 (2000) [hep-ph/0002033].

[26] S. Y. Choi, M. Guchait, H. S. Song and W. Y. Song, hep-ph/0007276.

[27] S. Y. Choi, J. Kalinowski, G. Moortgat-Pick and P. M. Zerwas, Eur. Phys. J. C 22, 563 (2001), Addendum-ibid. C 23, 769 (2002) [hep-ph/0108117]. 
[28] V. D. Barger, T. Falk, T. Han, J. Jiang, T. Li and T. Plehn, Phys. Rev. D 64, 056007 (2001) [hep-ph/0101106].

[29] A. Bartl, H. Fraas, O. Kittel and W. Majerotto, Phys. Rev. D 69 (2004) 035007 [hep$\mathrm{ph} / 0308141]$.

[30] A. Bartl, T. Kernreiter and O. Kittel, Phys. Lett. B 578, 341 (2004) [hep-ph/0309340].

[31] S. Y. Choi, M. Drees, B. Gaissmaier and J. Song, Phys. Rev. D 69, 035008 (2004) [hepph/0310284].

[32] A. Bartl, H. Fraas, O. Kittel and W. Majerotto, hep-ph/0402016.

[33] S. Y. Choi, M. Drees and B. Gaissmaier, hep-ph/0403054.

[34] A. Bartl, T. Kernreiter and W. Porod, Phys. Lett. B 538, 59 (2002) [hep-ph/0202198].

[35] A. Bartl, K. Hidaka, T. Kernreiter and W. Porod, Phys. Lett. B 538 (2002) 137 [hepph/0204071].

[36] A. Bartl, K. Hidaka, T. Kernreiter and W. Porod, Phys. Rev. D 66 (2002) 115009 [hepph/0207186].

[37] A. Bartl, H. Fraas, T. Kernreiter and O. Kittel, Eur. Phys. J. C 33 (2004) 433 [hep$\mathrm{ph} / 0306304]$.

[38] A. Bartl, S. Hesselbach, K. Hidaka, T. Kernreiter and W. Porod, Phys. Lett. B 573 (2003) 153 [hep-ph/0307317].

[39] A. Bartl, S. Hesselbach, K. Hidaka, T. Kernreiter and W. Porod, hep-ph/0311338.

[40] S. Y. Choi, K. Hagiwara and J. S. Lee, Phys. Rev. D 64 (2001) 032004 [hep-ph/0103294];

[41] A. Arhrib, D. K. Ghosh and O. C. W. Kong, Phys. Lett. B 537 (2002) 217 [hep$\mathrm{ph} / 0112039]$.

[42] S. Y. Choi, M. Drees, J. S. Lee and J. Song, Eur. Phys. J. C 25, 307 (2002) [hepph/0204200].

[43] M. Carena, J. R. Ellis, S. Mrenna, A. Pilaftsis and C. E. M. Wagner, Nucl. Phys. B 659 (2003) 145 [hep-ph/0211467].

[44] F. Borzumati, J. S. Lee and W. Y. Song, hep-ph/0401024.

[45] E. Christova, H. Eberl, W. Majerotto and S. Kraml, Nucl. Phys. B 639 (2002) 263, Erratum-ibid. B 647 (2002) 359 [hep-ph/0205227]; E. Christova, H. Eberl, W. Majerotto and S. Kraml, JHEP 0212 (2002) 021 [hep-ph/0211063].

[46] M. M. Nojiri, Phys. Rev. D 51 (1995) 6281 [hep-ph/9412374].

[47] M. M. Nojiri, K. Fujii and T. Tsukamoto, Phys. Rev. D 54, 6756 (1996) [hep-ph/9606370].

[48] E. Boos, H. U. Martyn, G. Moortgat-Pick, M. Sachwitz, A. Sherstnev and P. M. Zerwas, Eur. Phys. J. C 30 (2003) 395 [hep-ph/0303110]. 
[49] LEPSUSYWG, ALEPH, DELPHI, L3 and OPAL experiments, note LEPSUSYWG/01-03, http://lepsusy.web.cern.ch/lepsusy

[50] J. A. Aguilar-Saavedra et al. [ECFA/DESY LC Physics Working Group Collaboration], TESLA Technical Design Report Part III: Physics at an $e^{+} e^{-}$Linear Collider, hep$\mathrm{ph} / 0106315$.

[51] A. Bartl, H. Eberl, S. Kraml, W. Majerotto, W. Porod and A. Sopczak, Z. Phys. C 76 (1997) 549 [hep-ph/9701336]; A. Bartl, H. Eberl, S. Kraml, W. Majerotto and W. Porod, Eur. Phys. J. directC 2 (2000) 6 [hep-ph/0002115].

[52] See, for instance, G. Belanger, F. Boudjema, A. Cottrant, R. M. Godbole and A. Semenov, Phys. Lett. B 519 (2001) 93 [hep-ph/0106275].

[53] For a review see: W. Majerotto, arXiv:hep-ph/0209137, and references therein.

[54] For a recent summary, see R. M. Godbole, S. Kraml, M. Krawczyk, D. J. Miller, P. Niezurawski and A. F. Zarnecki, hep-ph/0404024.

[55] SUSY Parameter Analysis (SPA) project, http://www-flc.desy.de/spa/ 